\title{
Neutron cross section measurements of elemental molybdenum and resonance parameter analysis
}

\author{
G. Leinweber, ${ }^{1, a}$, D.P. Barry ${ }^{1}$, R.C. Block ${ }^{2}$, J.A. Burke (ret.) ${ }^{1}$, Y. Danon ${ }^{2}$, N.J. Drindak ${ }^{1}$, and B.E. Moretti ${ }^{3}$ \\ ${ }^{1}$ Lockheed Martin Corporation, P.O. Box 1072, Schenectady, New York 12301-1072, USA \\ 2 Gaerttner Laboratory, Rensselaer Polytechnic Institute, Troy, New York 12180-3590, USA \\ 3 United States Military Academy, Department of Physics, West Point, New York 10996, USA
}

\begin{abstract}
The purpose of this work was to measure the neutron cross sections of molybdenum accurately. The Rensselaer Polytechnic (RPI) LINAC facility was used to measure the neutron interaction cross sections of molybdenum. Neutron capture time-of-flight measurements were made at $25 \mathrm{~m}$ with a sodium iodide multiplicity detector. Transmission measurements were performed at $25 \mathrm{~m}$ flight with a ${ }^{6} \mathrm{Li}$ glass scintillation detector. Nine different thicknesses of elemental molybdenum metal samples ranging from $0.051 \mathrm{~mm}(0.002 \mathrm{in}$.) to $6.35 \mathrm{~mm}$ (0.250 in.) were measured in either capture or transmission. Data from two transmission and one capture measurement have been analyzed using the multilevel R-matrix Bayesian code SAMMY. Throughout the energy spectrum, 10$2000 \mathrm{eV}$, resonance widths have been attained. Between one and two $\mathrm{keV}$, the width assignments of overlapping resonances were obtained and compared to ENDF/B-VII.0. ENDF/B-VII.0 nuclear radii fit the transmission data between resonances better than those of ENDF/B-VI.8. Below $600 \mathrm{eV}$, the inclusion of capture data in the fit enhanced our ability to determine radiation widths compared to using transmission data alone.
\end{abstract}

\section{Introduction}

Initial transmission measurements were made in 1995 and preliminary results up to $200 \mathrm{eV}$ were reported [1]. Neutron capture data taken in 1995 have been included in the present analysis. In 2004 an additional transmission measurement was performed in order to extend the energy range of the analysis up to $2 \mathrm{keV}$. This latest measurement included thick $(>2.5 \mathrm{~mm})$ molybdenum and uranium samples capable of quantifying background, resolution function, and weak molybdenum resonances.

The RPI LINAC is a $60 \mathrm{MeV}$, pulsed, electron, linear accelerator. This machine has been used in conjunction with neutron-producing targets to make these measurements [1-3].

A review of the prior measurements shows important measurements of molybdenum published by Kapchigashev and Popov [4,5], Mitzel and Plendl [6], and Wynchank [7] below $1 \mathrm{keV}$. Measurements by Fricke et al. [8] and Weigmann [9] contributed above $1 \mathrm{keV}$.

\section{Experimental conditions}

The burst width was $100 \mathrm{~ns}$ for the capture experiment and $50 \mathrm{~ns}$ for the transmission experiments. Channel widths were $125 \mathrm{~ns}$ or less in the region of interest. The neutron energy for a detected event is determined using the time-of-flight (TOF) technique. The data acquisition system is described in refs. $[10,11]$. The beam and sample diameters were approximately $5 \mathrm{~cm}$. The capture detector is a sodium iodide scintillation detector in the form of an annulus around the neutron beam with the sample at its center [10-12].

${ }^{a}$ Presenting author, e-mail: leinwg@rpi .edu
Neutron transmission measurements were conducted at the 25.6-meter flight station. The detector consists of a $12.70-\mathrm{cm}$ (5-in) diameter, 1.27 -cm-thick NE $905{ }^{6} \mathrm{Li}$ glass scintillator and two photomultipliers placed outside of the neutron beam [13].

\section{Data reduction}

Neutron capture data taking and data reduction techniques at the RPI LINAC are described in refs. $[14,15]$. Processed capture data are expressed as yield. Yield is defined as the number of neutron captures per neutron incident on the sample.

In the 2004 transmission data, there were three black resonances in two molybdenum samples and five black resonances in two depleted uranium samples. These were used to fit the time-dependent background in the data. Transmission open beam background was estimated using the shape determined from the black molybdenum resonances and was normalized to a fixed sodium notch at $2.787 \mathrm{keV}$.

\section{Analysis method}

Resonance parameters neutron width, $\Gamma_{n}$, radiation width, $\Gamma_{\gamma}$, and resonance energy, $\mathrm{E}_{0}$, were extracted from the capture and transmission data sets using the multi-level R-matrix Bayesian code SAMMY version 7 [16]. This was a combined transmission and capture analysis which employed the resolution broadening, self-shielding, and multiple-scattering features of SAMMY. The efficiency of the capture detector to resonances in different molybdenum isotopes was investigated by fitting the flux normalization to resonances of different isotopes. The adequacy of using the same efficiency for all isotopes was determined to be correct to within $1.5 \%$. 


\section{Results}

An overview of the SAMMY fit in the epithermal region is given in figure 1 . Only one transmission and one capture data set are shown for clarity. The SAMMY curve is calculated from resonance parameters obtained from the combined transmission and capture, multi-sample fit.

Three experiments were performed to resolve molybdenum resonances below $200 \mathrm{eV}$. Overall, twelve samples were measured in transmission and capture.

Both capture and transmission data have been fitted to a single set of resonance parameters in the energy region below $600 \mathrm{eV}$. Neutron capture data were analyzed only below $600 \mathrm{eV}$ to minimize the effect of scattered neutrons entering the sodium iodide.

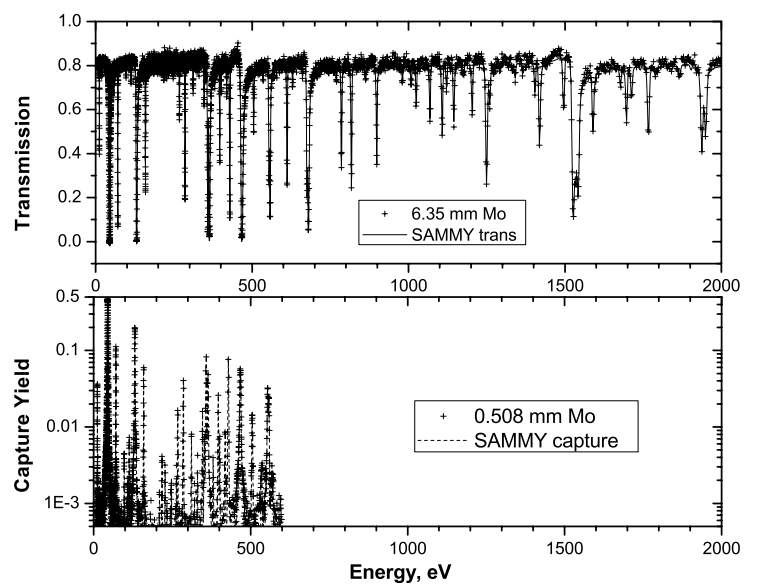

Fig. 1. The 2004 transmission measurement included molybdenum of two thicknesses; the $6.35 \mathrm{~mm}$ data are shown. The capture measurement included 4 thicknesses of molybdenum. All samples were elemental molybdenum. The SAMMY curves are the results of the combined transmission and capture, multi-sample fit.

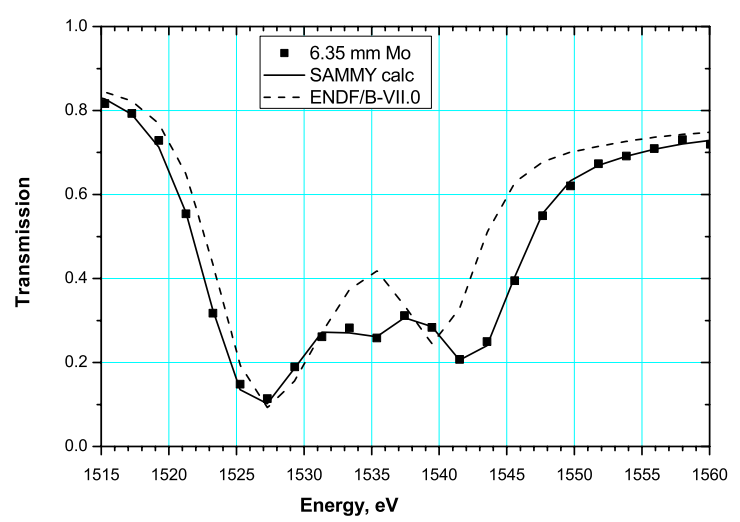

Fig. 2. Data and the SAMMY fit near $1535 \mathrm{eV}$ triplet. The data show a different distribution of resonance widths than those of ENDF.

Several significant contributions to the knowledge of molybdenum resonance parameters have been observed. A few examples occur at $131 \mathrm{eV}, 360 \mathrm{eV}, 680 \mathrm{eV}, 1250 \mathrm{eV}$, and $1535 \mathrm{eV}$ (fig. 2).
Throughout the energy spectrum improvements to resonance parameters were evident. A triplet of resonances at $1535 \mathrm{eV}$ is shown in figure 2. This region is an excellent example of where width assignments of overlapping resonances were improved over those of ENDF/B-VII.0 [17].

\section{Conclusions}

Elemental molybdenum was measured at the RPI LINAC facility. Resonance parameters were extracted from combined capture and transmission data sets using the multilevel R-matrix Bayesian code SAMMY. The analysis included Doppler broadening, resolution broadening and multiple scattering correcting of capture data. Separate resolution functions for transmission and capture were used.

The present measurements assumed the same spin assignments as ENDF/B-VII.0 for all resonances. The potential scattering radii from ENDF/B-VII.0 match the measured data much better than those of ENDF/B-VI.8. The resonance parameters from ENDF/B-VII.0, in general, match the measured data better than those of ENDF/B-VI.8. The resonance parameters from this measurement and analysis should improve the ENDF library.

\section{References}

1. B.E. Moretti, Ph.D. thesis, Rensselaer Polytechnic Institute, 1996.

2. R.W. Hockenbury et al., Phys. Rev. 178, 123 (2004).

3. M.E. Overberg et al., Nucl. Instrum. Meth. Phys. Res. A 438, 253 (1999).

4. S.P. Kapchigashev, Y.U. Popov, Joint Inst. For Nucl. Res., Dubna. Reports 1845, 104 (1964).

5. S.V. Kapchigashev, Y.U. Popov, Soviet Atomic Energy 15, 808 (1964).

6. F. Mitzel, H.S. Plendl, Nukleonik 6, 371 (1964).

7. S. Wynchank et al., Phys. Rev. 166, 4 (1968).

8. M.P. Fricke et al., Proc. 3rd Conf. on Neutron Cross-Sections and Technology, Univ. of Tennessee, Knoxville, 15-17 March 1971.

9. H. Weigmann et al., J. Nucl. Phys. Sect. A 104, 513 (1967).

10. R.E. Slovacek et al., Proc. Topl. Mtg. Advances in Reactor Physics, April 11-15, 1994, Knoxville, Tennessee.

11. R.C. Block et al., Proc. Int. Conf. Nuclear Data for Science and Technology, May 9-13, 1994, Gatlinburg, Tennessee, Vol. 1, American Nuclear Society, p. 81.

12. R.C. Block et al., Proc. Int. Conf. Nuclear Data for Science and Technology, May 30-June 3, 1988, Mito, Japan, p. 383.

13. D.P. Barry, Ph.D. thesis, Rensselaer Polytechnic Institute, 2003.

14. G. Leinweber et al., Nucl. Sci. Eng. 142, 1 (2002).

15. G. Leinweber et al., Nucl. Sci. Eng. 134, 50 (2000).

16. N.M. Larson, ORNL/TM-9179/R7, Oak Ridge National Laboratory (2006).

17. M.B. Chadwick et al., Nucl. Data Sheets 107(12), 2931 (2006). 